Meta

Journal des traducteurs

Translators' Journal

\title{
Interprétation ou interprétariat ?
}

Danica Seleskovitch

Volume 30, numéro 1, mars 1985

Interprétation de conférence

URI : https://id.erudit.org/iderudit/004086ar

DOI : https://doi.org/10.7202/004086ar

Aller au sommaire du numéro

Éditeur(s)

Les Presses de l'Université de Montréal

ISSN

0026-0452 (imprimé)

1492-1421 (numérique)

Découvrir la revue

Citer cet article

Seleskovitch, D. (1985). Interprétation ou interprétariat ? Meta, 30(1), 19-24.

https://doi.org/10.7202/004086ar d'utilisation que vous pouvez consulter en ligne.

https://apropos.erudit.org/fr/usagers/politique-dutilisation/ 


\section{INTERPRÉTATION OU INTERPRÉTARIAT?}

\section{SELESKOVITCH}

De tous temps et partout, celui qui savait plusieurs langues servait de truchement à ceux qui n'en savaient qu'une. Cela n'allait pas toujours sans danger puisque les vieilles chroniques rapportent qu'il arrivait aux interprètes d'être châtiés de leur double langage et de se voir arracher la langue pour les punir d'en avoir deux. Les interprètes modernes n'ont pas à craindre pareil châtiment, même si on en fait parfois mesquinement des boucs émissaires pour expliquer tel malentendu ou pour se dédire de telle parole hâtive ; mais le "double langage " désigne toujours la duplicité et la fourberie, de même qu'interpréter signifie souvent de nos jours être arbitraire ou tendancieux.

Parfois vilipendée, l'interprétation de conférence est néanmoins reconnue depuis plusieurs dizaines d'années comme un véritable métier, indispensable à la vie internationale. Son appellation l'est moins. Faut-il réellement dire interprétation ? Certains s'obstinent à parler d'interprétariat. Apparemment de pure forme, la différence est fondamentale car derrière le choix du mot se profilent des attitudes fort différentes.

Le terme interprétation existe depuis longtemps en français puisque le Petit Robert en fait remonter la première apparition à l'an 1160 . Il lui donne le sens de action d'expliquer, de donner une explication claire à une chose obscure. Y a-t-il plus obscur qu'un discours en une langue inconnue, peut-on lui donner une signification plus claire qu'en le restituant dans une langue connue ? Les interprètes de conférence qui fondèrent leur association internationale en 1953 ne s'y trompèrent pas : il ne fut jamais question pour eux de désigner leur activité d'un autre terme que celui d'interprétation.

Pourquoi alors le barbarisme interprétariat que rien ne justifie étymologiquement, car s'il est normal que notaire donne notariat ou secrétaire secrétariat, interprète devrait tout au plus donner interprétat ? Le Robert admet néanmoins ce barbarisme, le définissant ainsi : fonction, carrière d'interprète et donne comme exemple école d'interprétariat sans tenir compte du fait que, des deux écoles d'interprètes les plus prestigieuses, celles des universités de Genève et de Paris, l'une s'intitule école d'interprétation et de traduction et l'autre comprend deux sections : interprétation et traduction.

La querelle de mots qui oppose les tenants d'interprétariat aux interprètes mérite qu'on s'y attarde un instant ; en effet, les conceptions qui motivent ces termes sont diamétralement opposées, et leurs conséquences infiniment plus graves qu'il n'apparaît de prime abord. Ramenée à sa plus simple expression la conception qui est à l'origine d'interprétariat est que pour traduire il suffit de connaître les deux langues en présence et de substituer l'une à l'autre. Cette conception, intuitive ou consciente, explique que la plupart des écoles dites d'interprètes sont des écoles de langues où l'interprétation est aussi absente que végétation sur la lune ; elle explique aussi la naïveté des premiers travaux sur la traduction automatique qui a fait dépenser en pure perte des millions de dollars. La méprise est grave sur le plan théorique car on ne comprendra le processus de la traduction en général et celui de l'interprétation en particulier que si l'on comprend le processus de l'émission et de la réception de paroles, et si l'on admet que toute parole se 
transforme en pensée chez celui qui la reçoit et que c'est bien cette pensée que transmet le traducteur et non le sémantisme ou la syntaxe de la langue originale. C'est à défendre, avec le mot, la conception de l'interprétation que je m'attacherai ici.

L'interprétation est pratiquée par les bons interprètes non comme une opération sur les langues mais comme une opération sur ce qui se dit à travers les langues; pour eux, il s'agit de "comprendre et d'expliquer", il ne s'agit pas de convertir une langue en une autre.

Si unanimes que soient leurs affirmations à cet égard, elles n'auraient de justification qu'empirique si une étude plus détaillée n'en avait été faite. Cette étude a débuté il y a une quinzaine d'années à l'école que j'ai aujourd'hui l'honneur de diriger, l'École supérieure d'interprètes et de traducteurs de l'Université Paris III. Jusque-là, le champ de la théorie de l'interprétation restait largement en friche ou labouré uniquement par les retombées des théories linguistiques de la traduction qui connaissaient depuis la Seconde Guerre mondiale un essor sans pareil, ambitionnant de découvrir la pierre philosophale qui permettrait la transmutation d'une langue en une autre et donnerait à la traduction automatique ses lettres de créance.

Jusque-là, seul le résultat de la traduction écrite, la traduction produit était observable, l'acte de traduire restait inaccessible aux chercheurs.

Les choses allaient changer avec l'introduction de l'interprétation-métier dans la vie contemporaine et avec l'avènement quasi simultané du magnétophone. L'équipe de recherche de l'ÉSIT a enregistré en réunions internationales de nombreux discours avec leurs interprétations. La bande magnétique qui retient les discours et leurs interprétations consécutives ou simultanées fournit des tranches de langage d'une absolue authenticité. En les analysant, on constate que l'interprétation rend compte de chaque manifestation de sens de la chaîne sonore originale, de chaque intonation, de chaque hésitation, de chaque souffle du discours. Traduction immédiate, elle ne subit de corrections que linéaires, aucune retouche n'efface la version première, aucun remords ne gomme ce qui a été dit. Traduction brute, elle révèle le processus qui l'engendre.

En prenant l'interprétation sous la loupe nous avons pu la suivre à travers ses tâtonnements et conclure du processus de l'interprétation au processus de la traduction, de la compréhension des discours manifestée à travers l'interprétation à la compréhension du langage en général.

Nos analyses ont pris l'étude du langage à rebours par rapport à celle qui en est faite habituellement puisque au lieu de partir d'une pensée pour suivre sa mise en expression, nous partons de la réception d'une expression par l'interprète, nous observons le cheminement de sa compréhension à travers l'écoute de l'interprétation, nous la voyons aboutir et pouvons attester de sa réussite par l'écoute en parallèle de sa production et du discours original.

Que l'interprète travaille en consécutive ou en simultanée, la chaîne sonore constitue son point de départ. Nous disons bien chaîne sonore et non pas langue. Il convient de le souligner car il y a souvent méprise sur ce point ; on a trop tendance à penser que l'on entend du français alors que l'on entend que des sons français, on conclut a priori que ces sons correspondent aux signifiés qui sont les leurs en langue, c'est-à-dire en dehors de toute situation de communication. L'interprétation démontre que les sons éveillent des réalités, des notions, des sens, des idées, peu importe le terme, alors que hors situation de communication ces mêmes sons n'éveillent que des concepts.

Or on ne traduit pas de la même façon les paroles porteuses de sens et les concepts linguistiques. Donnons-en tout d'abord une démonstration, nous verrons ensuite comment le phénomène s'explique. 
Nous emprunterons notre exemple à Riesbeck et $S_{\text {chank }}{ }^{1}$ qui, pour démontrer que " to talk about the meaning of a sentence out of context is a nice abstract linguistic exercise, but it has little to do with how people actually function", allongent un même énoncé en le faisant précéder de différentes phrases; ils lui donnent ainsi ce que nous avons appelé des contextes cognitifs, qui le font passer de son sémantisme initial à plusieurs sens différents. L'interprète, pour qui la phrase antérieure devient savoir déverbalisé auquel se joint le sémantisme de l'énoncé actuel, restitue chaque fois le sens pertinent de l'énoncé.

La phrase prononcée est : I just came from New York. Hors contexte cognitif, prise telle quelle, elle a un sémantisme constitué de l'alignement syntaxique de concepts, qui peuvent se transcoder ainsi : Je viens d'arriver de New York. Ajoutons en amont les questions auxquelles les auteurs font répondre I just came from New York, et voyons les différentes versions qu'appelle la prise en compte de la première phrase dans l'interprétation de la seconde.

En réponse à Would you like to go to New York today?, I just came from New York! donnera J'en viens!

En réponse à Would you like to go to Boston today ?, I just came from New York.! donnera Je rentre à peine de New York!

En réponse à Why do you seem so out of place?, I just came from New York! donnera Je débarque, je suis New-Yorkais!

L'étude de l'interprétation permet ainsi de vérifier que les sens qui s'attachent aux sons de la chaîne parlée ne recoupent pas intégralement les concepts linguistiques et se traduisent donc différemment. cepts.

Encore s'agit-il d'en expliquer les raisons et de définir le sens par rapport aux con-

La psychologie nous apprend que toute perception, auditive, visuelle ou tactile, provient de l'interprétation d'une sensation, c'est-à-dire de la relation qui s'établit à notre insu entre une information sensorielle actuelle et une autre ou d'autres informations actuelles ou antérieures. L'information sensorielle, la sensation, c'est-à-dire la partie effectivement appréhendée par les sens et structurée en fonction d'expériences antérieures pour identifier ce dont il s'agit. Ainsi ne voit-on jamais en même temps qu'une partie d'un chat mais on identifie l'animal qu'on l'ait vu de dos, de face ou de côté, en train de se lécher ou de dormir, et on sait qu'il s'agit de Pénélope lorsque, grâce à des connaissances supplémentaires on reconnaît, au-delà de l'espèce, le spécimen. On ne la gronde, cependant, ou on ne la caresse qu'en fonction de la situation unique appréhendée hic et nunc.

Qu'en est-il de la chaîne sonore ? Information sensorielle, elle est structurée de façon à faire surgir des signifiants ; on les identifie dans une langue que l'on connaît et on leur attribue sans mal les significations qui s'y attachent ; indépendamment de toute situation de communication on reconnaît des mots et des phrases derrière les signifiants que l'on identifie. Les phrases dont la composition est inédite, les phrases jamais entendues précédemment, sont reconnues elles aussi à partir de leurs seules sonorités.

Mais en situation de communication, l'énoncé linguistique produit chez l'auditeur une réaction cognitive qui dépasse la valeur des mots, car chaque parole, chaque unité de discours est tributaire de ce qui a précédé et de ce qui suit et, s'insérant dans l'expérience antérieure de l'auditeur, se charge de sens qui sont insoupçonnables au niveau de l'analyse linguistique de phrases hors situation.

Chacun sait que, à force de nommer TABL d'innombrables tables réelles on en induit le concept de table, proposé par la langue maternelle. Cette acquisition de concepts, produits de la conjugaison de la langue et de l'expérience de l'individu, donne à 
l'homme adulte ayant acquis la compétence de sa langue maternelle d'infinies possibilités d'expression. Mais virtualité n'est pas réalité. Grâce à l'interprétation on peut constater que les concepts qui caractérisent la langue au repos s'enrichissent considérablement dans son emploi. L'interprétation montre que si, en langue, les sonorités signifient conceptuellement, employées dans le discours elles signalent des sens qui vont bien audelà. En l'observant, on constate un fait somme toute banal mais rarement noté, à savoir que la mise en œuvre de sons dans la parole vise des réalités physiques ou psychiques. L'existence de concepts n'y change rien.

En s'enrichissant en situation de communication, en désignant des réalités au-delà des concepts, les structures sonores perdent du même coup la plupart de leurs strates sémantiques pour n'en garder qu'une. La parole n'éveille pas toutes les virtualités qui s'attachent de façon stable aux structures sonores de la langue ; elle ne connaît ni polysémie ni ambiguites. Coat dans this dog has a heavy coat sera toujours compris comme poil ou fourrure et non comme manteau; I bought a chair at the furniture store ne sera pas confondu avec chaire en Sorbonne. Beaucoup plus que d'une désambiguïsation, il s'agit là d'une actualisation de traits sémantiques pertinents en raison des contextes verbal et cognitif. Les sens des discours sont ainsi perçus grâce à la jonction d'actualisations sémiques à des savoirs antérieurs.

Ce sera toujours l'association des énoncés à des connaissances antérieures qui produira des sens inédits et donc des connaissances nouvelles. Comme le dit E.M. Uhlenbeck : "Meaning is knowledge functioning in and during the use of language ${ }^{2}$. Ce sens inédit s'intègre dans le savoir antérieur, la perception du discours progressant par vagues de savoirs intégrés en nouveaux sens inédits.

Ces sens étant plus vastes que les concepts, on comprend qu'ils se traduisent par les traits les plus aptes à les faire comprendre et non par la juxtaposition des concepts correspondants.

La notion de sens opposée à sémantisme repose sur une observation minutieuse des faits de parole et d'interprétation ; il s'agit là d'un constat établi quotidiennement. On nous oppose néanmoins parfois deux séries d'objections qui, sans se préoccuper des faits, se cantonnent dans l'argumentation abstraite. Nous voudrions leur répondre ici. Elles portent sur la fidélité au discours et à travers lui à son auteur. N'y a-t-il pas abus à vouloir interpréter, disent les uns, le transcodage, l'interprétariat n'est-il pas plus fidèle que l'interprétation ? S'il est vrai, disent les autres, que l'interprétation est dans la nature des choses, ne doit-on pas craindre ses débordements et où tracer les limites de sa liberté ?

Nous répondrons tout d'abord que l'interprétation de conférence doit prendre pour objet le sens parce que le transcodage est incapable de transmettre un discours. Voyons pourquoi.

Nous avons appelé transcodage la traduction qui ne reposerait que sur une double compétence linguistique ; ce type de traduction devrait être exécuté sans recours aucun au bagage cognitif que chaque traducteur a accumulé en plus de l'acquisition de ses deux langues ; il devrait être exécuté sans qu'intervienne une quelconque prise de conscience du sens des paroles échangées, des mobiles de ceux qui parlent ou de la situation où ils se trouvent. Ne mettant en jeu que les deux langues en présence, il connaîtrait tous les problèmes qui hantent la traduction automatique depuis des décennies et notamment le problème d'ambiguité : ambiguités de segmentation (à Miami ou ami, ami ? les petits trous de la locomotive ou les petites roues de la locomotive ?) ambiguïtés de catégorie grammaticale (clean $=$ propre ou nettoie $?$, flying planes can be dangerous $=$ les avions en vol risquent d'être dangereux ou piloter des avions est dangereux ?), ambiguïtés syntaxiques (primary energy consumption = consommation primaire d'énergie ou con- 
sommation d'énergie primaire ? First election results = résultats des premières élections oụ premiers résultats des élections ? etc. Comment choisir entre des virtualités sémantiques en l'absence de critères cognitifs ?

À ces ambiguïtés révélées par l'analyse linguistique s'ajoute la polysémie des signifiants dont chacun doit être considéré comme retenant un ensemble de traits de signification ou sèmes qui font de chaque syntagme un ensemble sémique. Interpréter en ne faisant appel qu'à la seule connaissance des langues serait imaginable si les deux langues en présence présentaient les mêmes ambiguïtés et se composaient d'ensemble sémiques identiques. Le Grand Robert donne pour le seul mot pied une quarantaine de définitions, de l'extrémité de la jambe aux figements métaphoriques du terme tels que ça me casse les pieds, c'est le pied, avoir un pied dans la tombe, etc., à avoir pied ou perdre pied, au pied du lit, au pied du mur, etc. Peut-on imaginer que dans toutes les langues, pied englobe toutes ces virtualités?

La traduction littérale, loin de laisser à l'auditeur le soin de désambiguïser et de faire choix de la signification pertinente des mots, n'est qu'opaque inintelligibilité. Comment traduire littéralement : he went to the store for mother? il alla au magasin pour mère ne signifie rien; il faut donc choisir entre il est allé faire une course pour maman ou il est allé chercher maman au magasin. La compétence linguistique suffit pour aligner les deux variantes, mais aucun critère linguistique ne permet de faire un choix entre l'un ou l'autre. Faudrait-il alors, s'interdisant de faire appel à une connaissance supplémentaire et présentement non verbalisée (le souvenir que la mère se trouve dans le magasin, par exemple), qui sortirait le transcodage de l'ornière et le ramènerait d'emblée à une interprétation, énumérer les différentes possibilités signifiantes ?. L'interdiction faite à l'intelligence et à la mémoire cognitive produirait des alignements intolérables de signifiés...

Ceux qui nous posent la deuxième série de questions admettent que l'interprétation doive transcender les signifiés mais craignent ses débordements. L'interprétation ne risque-t-elle pas de transformer l'interprète en commentateur ou en exégète ? Où tracer les limites de sa liberté, si elles ne sont plus données par le sémantisme linguistique?

Ces critiques ont raison de poser la question, mais leurs craintes ne sont pas justifiées ; il n'y a pas à craindre d'excès de liberté, car les sens ont des attaches aussi solides et aussi démontrables avec les signifiants des paroles, que les signifiés avec ces mêmes signifiants hors situation. L'exemple le plus banal en est le je ou le vous, qui ne prennent leur sens que lorsque je se met à parler en s'adressant à vous ; ce je et ce vous sont alors aussi nettement délimités au niveau des réalités que le sont les pronoms au plan de la langue.

Si conserver le sens exige une interprétation, l'interprète n'excipe pas de cet acte d'intelligence pour aller au-delà de sa fonction. Les limites de l'interprétation sont claires, elles n'autorisent aucune hypothèse sur l'intention d'un orateur, jamais en tout cas l'expression de cette hypothèse. Les intentions des orateurs, l'objectif poursuivi par leurs interventions - vouloir plaire ou au contraire menacer, flatter, concéder, faire rire, convaincre - sous-tendent leurs dires sans être verbalisées. L'interprète les capte avec plus ou moins de certitude mais il n'est pas question pour lui de les exprimer.

Les théoriciens du " langage comme action ", et en particulier Austin, ont étudié les formes linguistiques qui impliquent une intention. Les mots $i l$ fait froid dits dans une relation sociale de supérieur à subordonné peuvent révéler l'intention de faire fermer une fenêtre ; dits dans un rapport inverse, ils peuvent signifier une demande d'autorisation de la fermer. L'énoncé : Vous fumez ? exprimera des intentions différentes selon que l'on imagine telle ou telle situation de discours ; dans une réunion de travail ou dans un 
train, l'intention peut être Voulez-vous une cigarette? ou Est-ce que la fumée vous dérange?

Le langage ne possède pas seulement un sémantisme - les significations en langue qui font l'objet de l'apprentissage des langues maternelle et étrangères; dans le discours il ne fournit pas seulement des sens, objets de l'interprétation, produits de la jonction entre des savoirs actualisés mais non verbalisés et le sémantisme présent ; il permet aussi d'indiquer des intentions - déterminantes pour les rapports entre les individus, objets de toutes les exégèses, de la psychanalyse à l'analyse des discours politiques ou à l'exégétique des textes sacrés.

Mais l'interprète, lui, se situe strictement au niveau des sens. Il ne se permettra pas de traduire Il fait froid par Fermez la fenêtre ou Vous fumez? par Est-ce que la fumée vous dérange ? Il lui faut, pour être clair, utiliser dans sa langue d'expression des formes linguistiques différentes de l'original, mais celles-ci désignent les mêmes notions, qu'on les appelle sens, idée ou réalité.

Alors interprétariat ou interprétation? Lorsque l'on constate l'extraordinaire complexité des langues comparé à la simplicité de leur emploi, on ne peut que s'étonner du paradoxe qu'il y a à croire à l'interprétariat et à craindre l'interprétation. Ne faire appel, pour traduire, qu'à la connaissance des langues, ne pas se soucier de ce que les mots signifient hic et nunc, c'est compliquer les discours à l'extrême en les réduisant à des alignements de concepts, c'est ne concevoir la traduction que comme la mise en regard d'autres concepts.

En fait, le transcodage est une chimère, une fiction irréalisable car contraire au fonctionnement du langage. Ceux qui s'y appliquent pour traduire ne parviennent qu'à produire des sons impuissants à évoquer le contenu original.

Interpréter pour traduire par contre, c'est donner à la traduction un caractère de normalité, c'est la sortir de l'artificialité de la langue, c'est lui restituer son objet véritable : les discours et les textes, c'est lui permettre de refléter le fonctionnement banal de toute communication langagière.

Accepter « interprétation » c'est poser correctement le phénomène de la traduction, c'est donner des fondements solides à son exercice, à son enseignement et à sa théorisation.

Notes

1. C.C. Riesbeck et R.C. Schank (1978) : "Comprehension by Computer : Expectation-Based Analysis of Sentences in Context", in W.J.M. Levelt et G.B. Flores-d'Arcais (eds.), Studies in the Perception of Language, John Wiley \& Sons, New York.

2. E.M. Uhlenbeck : "Observation in Semantics is not Easy", in D.J. van Alkemade (et al. (eds.) : Linguistic Studies, p. 134.

\section{RÉFÉRENCES}

DELISLE, Jean (1980) : l'Analyse du discours comme méthode de traduction, Éditions de l'Université d'Ottawa.

DELISLE, Jean (éd.) (1981) : Collectif ÉTI (Ottawa)/ÉSIT (Paris) : l'Enseignement de la traduction et de l'interprétation, Éditions de l'Université d'Ottawa.

LEDERER, Marianne (1981) : la Traduction simultanée, fondements théoriques, Minard, Paris.

SELESKOVITCH, Danica (1968) : l'Interprète dans les conférences internationales, Minard (traductions anglaise, espagnole et chinoise).

SELESKOVITCH, Danica (1975) : Langage, langues et mémoire, Minard.

SELESKOVITCH, Danica et Marianne LEDERER (1984) : Interpréter pour traduire, Didier Érudition. 